\title{
The space of stability conditions on the projective plane
}

\author{
Chunyi Li ${ }^{1}$
}

\begin{abstract}
The space of Bridgeland stability conditions on the bounded derived category of coherent sheaves on $\mathbf{P}^{2}$ has a principal connected component $\operatorname{Stab}^{\dagger}\left(\mathbf{P}^{2}\right)$. We show that $\operatorname{Stab}^{\dagger}\left(\mathbf{P}^{2}\right)$ is the union of geometric and algebraic stability conditions. As a consequence, we give a cell decomposition for $\operatorname{Stab}^{\dagger}\left(\mathbf{P}^{2}\right)$ and show that $\operatorname{Stab}^{\dagger}\left(\mathbf{P}^{2}\right)$ is contractible.
\end{abstract}

Keywords Stability condition - Exceptional collection $\cdot$ Cell decomposition

Mathematics Subject Classification 14F05 - 18E30

\section{Introduction}

Motivated by the concept of $\Pi$-stability condition on string theory by Douglas, the notion of a stability condition, $\sigma=(\mathcal{P}, Z)$, on a $\mathbb{C}$-linear triangulated category $\mathcal{T}$ was first introduced by Bridgeland in [8]. In the notion, the central charge $Z$ is a group homomorphism from the numerical Grothendieck group $\mathrm{K}_{0}(\mathcal{T})$ to $\mathbb{C}$. Bridgeland proves that the space of stability conditions inherits a natural complex manifold structure via local charts of central charges in $\operatorname{Hom}_{\mathbb{Z}}\left(\mathrm{K}_{0}(\mathcal{T}), \mathbb{C}\right)$. In particular, when $\mathrm{K}_{0}(\mathcal{T})$ has finite rank, the space of stability condition (satisfying support condition), $\operatorname{Stab}(\mathcal{T})$, has complex dimension $\operatorname{rank}\left(\mathrm{K}_{0}(\mathcal{T})\right)$.

As mentioned in [10], $\operatorname{Stab}(\mathcal{T})$ is expected to be related to the study of string theory and mirror symmetry. The main interesting example is to understand the space

$凶$ Chunyi Li

Chunyi.Li@ed.ac.uk

1 School of Mathematics, The University of Edinburgh, James Clerk Maxwell Building, The King's Buildings, Mayfield Road, Edinburgh EH9 3JZ, Scotland, UK 
of stability conditions on a compact Calabi-Yau threefold $X$ such as a quintic in $\mathbf{P}^{4}$. Yet this problem is still wildly open mainly due to some technical difficulties. Although the compact Calabi-Yau threefold case is still difficult to study, $\operatorname{Stab}(\mathcal{T})$ of various analogous categories has been very well understood, see [7,11,13,16,23]. While most of these examples are built from quivers or locally derived category of subvarieties, few cases of $\operatorname{Stab}(X)$ for smooth compact varieties $X$ are known. Such $\operatorname{Stab}(X)$ is 'well-understood' only when $X$ is $\mathbf{P}^{1}([21])$, a curve ([8]), a K3 surface ([3,9]), an abelian surface or threefold ([5]). In this paper, based on some important technical results from [19] and [20], we make an attempt to analyze the space $\operatorname{Stab}\left(\mathbf{P}^{2}\right)$.

Theorem 0.1 (Theorem 3.9, Corollary 3.10) Let $\mathrm{Stab}^{\dagger}\left(\mathbf{P}^{2}\right)$ be the connected component in $\operatorname{Stab}\left(\mathbf{P}^{2}\right)$ that contains the geometric stability conditions, then

$$
\operatorname{Stab}^{\dagger}\left(\mathbf{P}^{2}\right)=\operatorname{Stab}^{\mathrm{Geo}}\left(\mathbf{P}^{2}\right) \bigcup \operatorname{Stab}^{\mathrm{Alg}}\left(\mathbf{P}^{2}\right)
$$

In particular, $\operatorname{Stab}^{\dagger}\left(\mathbf{P}^{2}\right)$ is contractible.

Here $\operatorname{Stab}^{\mathrm{Geo}}(X)$ denotes the space of geometric stability conditions (Definition $1.7)$, at which the sky-scraper sheaves are stable with a same phase. $\operatorname{Stab}^{\mathrm{Alg}}\left(\mathbf{P}^{2}\right)$ denotes the space of algebraic stability conditions (Definition 2.3), which can be constructed from exceptional collections.

\section{Description for $\operatorname{Stab}^{\dagger}\left(\mathbf{P}^{2}\right)$}

We first describe the geometric part $\operatorname{Stab}^{\mathrm{Geo}}(X)$. When $X$ is a smooth surface, by [3] and [9], $\operatorname{Stab}^{\mathrm{Geo}}(X)$ can be determined if one knows the Chern characters of Giesekerstable sheaves. The $\tilde{G L}^{+}(2, \mathbb{R})$-action (see Lemma 8.2 in [8]) is free on the part of $\operatorname{Stab}^{\mathrm{Geo}}(X)$. Any point in $\mathrm{Stab}^{\mathrm{Geo}}(X) / \tilde{\mathrm{GL}}^{+}(2, \mathbb{R})$ is uniquely determined by the kernel of its central charge, which is a linear subspace in $\mathrm{K}_{\mathbb{R}}(X)$ of real codimension two. Conversely, such a linear subspace can be realized as the kernel of a central charge if and only if one can construct a quadratic form $Q$ on $\mathrm{K}_{\mathbb{R}}(X)$ satisfying the support condition (see the definition above Definition 1.7) for this subspace. In the case that $X$ is of Picard number one, one may take the projectivization of $\mathrm{K}_{\mathbb{R}}(X)$, Ker $Z$ is a point on $\mathrm{P}\left(\mathrm{K}_{\mathbb{R}}(X)\right)$. A point in $\mathrm{P}\left(\mathrm{K}_{\mathbb{R}}\left(\mathbf{P}^{2}\right)\right)$ can be the kernel of a central charge if and only if it has an open neighborhood which is not 'below' any Gieseker stable character.

Now we focus on the case that $\mathcal{T}$ is $\mathrm{D}^{b}\left(\mathbf{P}^{2}\right)$. The (projective) Gieseker stable characters have been completely determined in [14] by Drezet and Le Potier. On $\mathrm{P}\left(\mathrm{K}_{\mathbb{R}}\left(\mathbf{P}^{2}\right)\right)$, the characters form a dense set below the Le Potier curve (see Definition 1.4) together with some isolated points of exceptional characters.

For the algebraic part $\operatorname{Stab}^{\mathrm{Alg}}\left(\mathbf{P}^{2}\right)$, it goes back to the work [6] that $\mathrm{D}^{b}\left(\mathbf{P}^{2}\right)$ can be generated by an exceptional collection $\{\mathcal{O}, \mathcal{O}(1), \mathcal{O}(2)\}$. One can do mutations between the exceptional objects to get other exceptional triples, such as $\left\{\mathcal{O}(1), \mathcal{T}_{\mathbf{P}^{2}}, \mathcal{O}(2)\right\},\{\mathcal{O}(-4), \mathcal{O}(-3), \mathcal{O}(-2)\}$, which also generates the category. For each exceptional triple $\mathcal{E}=\left\langle E_{1}, E_{2}, E_{3}\right\rangle$, one may assign numbers $z_{j}=$ $m_{j} \exp \left(i \pi \phi_{j}\right), \phi_{j}$ as the central charges and phases of $E_{j}$. Due to the result in [20], 
when $m_{j} \in \mathbb{R}_{>0}, \phi_{1}<\phi_{2}<\phi_{3}$, and $\phi_{1}+1<\phi_{3}$, there is a unique stability condition with the given central charge and $E_{i} \in \mathcal{P}\left(\phi_{i}\right)$. Denote all such stability conditions by $\Theta_{\mathcal{E}}$ with parameters $m_{j}$ and $\phi_{j}$. The space of algebraic stability conditions $\operatorname{Stab}^{\mathrm{Alg}}\left(\mathbf{P}^{2}\right)$ is the union of all $\Theta_{\mathcal{E}}$. Note that the $\tilde{\mathrm{GL}}^{+}(2, \mathbb{R})$-action is not free on $\operatorname{Stab}^{\mathrm{Alg}}\left(\mathbf{P}^{2}\right)$. Each $\Theta_{\mathcal{E}}$ can be divided into three parts: the head $\Theta_{\mathcal{E}}^{\mathrm{Geo}}$; the legs $\Theta_{\mathcal{E}, E_{1}}^{+}, \Theta_{\mathcal{E}, E_{3}}^{-}$; and the tail $\Theta_{\mathcal{E}}^{\text {Pure }}$ (see Definition 2.3). The head part is the overlap part with the geometric stability conditions, this is the only part of $\Theta_{\mathcal{E}}$ that 'glues' on the $\operatorname{Stab}^{\mathrm{Geo}}(X)$. The leg part overlaps with other algebraic stability conditions, we will show that any two legs of $\Theta_{\mathcal{E}}$ and $\Theta_{\mathcal{E}^{\prime}}$ are either the same, or separated from each other (see Proposition 3.4). Each tail part $\Theta_{\mathcal{E}}^{\text {Pure }}$ is a private area for $\Theta_{\mathcal{E}}$, which is separated from any other $\Theta_{\mathcal{E}^{\prime}}$ (see Lemma 2.4). We will show that one may contract the whole space of $\operatorname{Stab}^{\mathrm{Alg}}\left(\mathbf{P}^{2}\right)$ by first contracting all the tails simultaneously to their boundaries with the legs, and then contracting all the legs to their boundaries with heads. The union of all heads $\bigcup \Theta_{\mathcal{E}}^{\text {Geo }}$ is a $\tilde{G L}^{+}(2, \mathbb{R})$-fiber space over an open subset of $\operatorname{Stab}^{\mathrm{Geo}}\left(\mathbf{P}^{2}\right)$, which is contractible.

\section{Related works}

Many important technical results on $\operatorname{Stab}^{\mathrm{Alg}}\left(\mathbf{P}^{2}\right)$ have been set up in [19] and [20], and our result is a natural continuation of the previous work. The space $\operatorname{Stab}^{\dagger}\left(\mathbf{P}^{2}\right)$ can be compared with some previous geometric examples such as $\mathrm{Stab}^{\dagger}(\mathrm{K} 3)$ and $\mathrm{Stab}^{\dagger}$ (local $\mathbf{P}^{2}$ ). As described in the previous section, their geometric parts $\operatorname{Stab}^{\mathrm{Geo}}(X)$ are quite similar. In addition, each exceptional/spherical object provides two boundary sets of $\mathrm{Stab}^{\mathrm{Geo}}(X)$. But the remaining parts are very different: for a $\mathrm{K} 3$ surface or local $\mathbf{P}^{2}$, the remaining parts can be viewed as copies of the geometric part. While for $\operatorname{Stab}^{\dagger}\left(\mathbf{P}^{2}\right)$, the remaining parts are similar to the space of stability conditions of quivers representations, see the works of $[7,11,13,16,23,24]$. In most of the previous quiver representation examples, the stability conditions are all of the algebraic type. Yet the quiver representation for $\mathrm{D}^{\mathrm{b}}\left(\mathbf{P}^{2}\right)$ has a complicated relation, this leads to the fact that some of the geometric stability conditions on $\mathbf{P}^{2}$ are not of the algebraic type. In addition, it seems to the author that the contractibility of the algebraic parts $\operatorname{Stab}^{\mathrm{Alg}}\left(\mathbf{P}^{2}\right)$ is not implied by the results in any of the previous papers. In particular, the paper [24], in which the authors proved the contractibility for many interesting examples, does not apply to the case of $\operatorname{Stab}^{\mathrm{Alg}}\left(\mathbf{P}^{2}\right)$, since the heart $\langle\mathcal{O}[2], \mathcal{O}(1)[1], \mathcal{O}(2)\rangle$ is not locally finite and has infinitely many algebraic tilts, which are crucial assumptions on the t-structure in [24].

\section{Open questions}

It is reasonable for us to believe that $\operatorname{Stab}^{\dagger}\left(\mathbf{P}^{2}\right)$ actually contains all the stability conditions that satisfy the support condition.

Conjecture 0.2 We expect the following statement holds: $\operatorname{Stab}\left(\mathbf{P}^{2}\right)=\operatorname{Stab}^{\dagger}\left(\mathbf{P}^{2}\right)$.

In addition, as in the case of $\mathbf{P}^{1}$, we wish to understand the global complex structure of $\operatorname{Stab}\left(\mathbf{P}^{2}\right)$. We expect that there is a period map as that of the CY quiver cases, $[11,16]$, 
so that we may have differential forms on $\operatorname{Stab}^{\dagger}\left(\mathbf{P}^{2}\right)$ and the central charge is neatly computed as integrations. But this seems difficult to realize because there is some 'pure geometric' part on $\operatorname{Stab}^{\dagger}\left(\mathbf{P}^{2}\right)$. For the algebraic part $\operatorname{Stab}^{\mathrm{Alg}}\left(\mathbf{P}^{2}\right)$, we also expect that there is a fundamental domain $R_{\mathcal{E}}$ on $(\mathcal{H})^{3} \simeq \Theta_{\mathcal{E}}$ independent of the triples $\mathcal{E}$ such that all the $R_{\mathcal{E}}$ 's form a disjoint cover of $\operatorname{Stab}^{\mathrm{Alg}}\left(\mathbf{P}^{2}\right)$.

\section{Notations}

The Picard group of $\mathbf{P}^{2}$ is of rank one with generator $H=[\mathcal{O}(1)]$, and we will, by the abuse of notation, identify the $i$ th Chern character $\mathrm{ch}_{i}$ with its degree $H^{2-i} \mathrm{ch}_{i}$. The slope $\mu$ of a non-torsion sheaf $E$ on $\mathbf{P}^{2}$ is defined as $\frac{\mathrm{ch}_{1}}{\mathrm{ch}_{0}}$. We denote $\mathrm{K}\left(\mathbf{P}^{2}\right) \otimes \mathbb{R}$ by $\mathrm{K}_{\mathbb{R}}\left(\mathbf{P}^{2}\right)$. Consider the real projective space $\mathrm{P}\left(\mathrm{K}_{\mathbb{R}}\left(\mathbf{P}^{2}\right)\right)$ with homogeneous coordinate [ $\mathrm{ch}_{0}, \mathrm{ch}_{1}, \mathrm{ch}_{2}$, we view the locus $\mathrm{ch}_{0}=0$ as the line at infinity. The complement forms an affine real plane, which is referred to as the $\left\{1, \frac{\mathrm{ch}_{1}}{\mathrm{ch}_{0}}, \frac{\mathrm{ch}_{2}}{\mathrm{ch}}\right\}$-plane. We call $\mathrm{P}\left(\mathrm{K}_{\mathbb{R}}\left(\mathbf{P}^{2}\right)\right)$ the projective $\left\{1, \frac{\mathrm{ch}_{1}}{\mathrm{ch}_{0}}, \frac{\mathrm{ch}_{2}}{\mathrm{ch}_{0}}\right\}-$ plane. For any object $F$ in $\mathrm{D}^{b}\left(\mathbf{P}^{2}\right)$, we write

$$
\tilde{v}(F):=\left(\operatorname{ch}_{0}(F), \operatorname{ch}_{1}(F), \operatorname{ch}_{2}(F)\right)
$$

as the numerical character of $F$, and $v(F)$ the projection of $\tilde{v}(F)$ on the $\left\{1, \frac{\mathrm{ch}_{1}}{\mathrm{ch}_{0}}, \frac{\mathrm{ch}_{2}}{\mathrm{ch}_{0}}\right\}$-plane with locus $(1, s, q)$.

Let $E, F$ be two objects in $\mathrm{D}^{b}\left(\mathbf{P}^{2}\right)$ with linear independent characters on the $\left\{1, \frac{\mathrm{ch}_{1}}{\mathrm{ch}_{0}}, \frac{\mathrm{ch}_{2}}{\mathrm{ch}}\right\}$-plane and $P$ be a point on the projective $\left\{1, \frac{\mathrm{ch}_{1}}{\mathrm{ch}_{0}}, \frac{\mathrm{ch}_{2}}{\mathrm{ch}_{0}}\right\}-$ plane. For the convenience of the reader, we make the list of notations and symbols that are commonly used in this article. Most of them are explicitly defined at other places of the article.

\section{Geometric stability conditions}

\subsection{Review: exceptional objects, triples, and Le Potier curve}

Let $\mathcal{T}$ be a $\mathbb{C}$-linear triangulated category of finite type. For convenience, one may always assume that $\mathcal{T}$ is $\mathrm{D}^{\mathrm{b}}\left(\mathbf{P}^{2}\right)$ : the bounded derived category of coherent sheaves on the projective plane over $\mathbb{C}$. The following definitions follow from $[2,15,22]$.

Definition 1.1 An object $E$ in $\mathcal{T}$ is called exceptional if

$$
\operatorname{Hom}^{i}(E, E)=0, \quad \text { for } i \neq 0 ; \operatorname{Hom}^{0}(E, E)=\mathbb{C} .
$$

An ordered collection of exceptional objects $\mathcal{E}=\left\{E_{0}, \ldots, E_{m}\right\}$ is called an exceptional collection if

$$
\operatorname{Hom}^{\bullet}\left(E_{i}, E_{j}\right)=0, \quad \text { for } i>j .
$$

Definition 1.2 Let $\mathcal{E}=\left\{E_{0}, \ldots, E_{n}\right\}$ be an exceptional collection. We call this collection $\mathcal{E}$ strong, if 


$$
\operatorname{Hom}^{q}\left(E_{i}, E_{j}\right)=0
$$

for all $i, j$ and $q \neq 0$. This collection $\mathcal{E}$ is called full, if $\mathcal{E}$ generates $\mathcal{T}$ under homological shifts, cones and direct summands.

We summarize some of the classification results of the exceptional bundles on $\mathbf{P}^{2}$ and make some notations, see $[14,15,17]$. There is a one-to-one correspondence between the dyadic integers $\frac{p}{2^{m}}$ and exceptional bundles $E_{\left(\frac{p}{2^{m}}\right)}$. Let the Chern character of the exceptional bundle corresponding to $\frac{p}{2^{m}}$ be

$$
\tilde{v}\left(E_{\left(\frac{p}{2^{m}}\right)}\right):=\left(\operatorname{ch}_{0}\left(E_{\left(\frac{p}{2^{m}}\right)}\right), \operatorname{ch}_{1}\left(E_{\left(\frac{p}{2^{m}}\right)}\right), \operatorname{ch}_{2}\left(E_{\left(\frac{p}{2^{m}}\right)}\right)\right)
$$

the characters are inductively given by the formulas:

- $\tilde{v}\left(E_{(n)}\right)=\left(1, n, \frac{n^{2}}{2}\right)$, for $n \in \mathbb{Z}$.

- When $m>0$ and $p \equiv 3(\bmod 4)$, the character is given by

$$
\tilde{v}\left(E_{\left(\frac{p}{2^{m}}\right)}\right)=3 \operatorname{ch}_{0}\left(E_{\left(\frac{p+1}{2^{m}}\right)}\right) \tilde{v}\left(E_{\left(\frac{p-1}{2^{m}}\right)}\right)-\tilde{v}\left(E_{\left(\frac{p-3}{2^{m}}\right)}\right) .
$$

- When $m>0$ and $p \equiv 1(\bmod 4)$, the character is given by

$$
\tilde{v}\left(E_{\left(\frac{p}{2^{m}}\right)}\right)=3 \operatorname{ch}_{0}\left(E_{\left(\frac{p-1}{2^{m}}\right)}\right) \tilde{v}\left(E_{\left(\frac{p+1}{2^{m}}\right)}\right)-\tilde{v}\left(E_{\left(\frac{p+3}{2^{m}}\right)}\right) .
$$

Example 1.3 Here are some first observations from the definition.

1. When $k \in \mathbb{Z}, \tilde{v}\left(E_{(k)}\right)$ is the character for the line bundle $E_{(k)}=\mathcal{O}_{\mathbf{P}^{2}}(k)$.

2. $\tilde{v}\left(E_{\left(\frac{3}{2}\right)}\right)$ is the character for the tangent bundle $E_{\left(\frac{3}{2}\right)}=\mathcal{T}_{\mathbf{P}^{2}}$.

3. The exceptional bundle $E_{\left(\frac{p}{2^{m}}+1\right)}$ associates to $\frac{p}{2^{m}}+1$ is $E_{\left(\frac{p}{2^{m}}\right)} \otimes \mathcal{O}_{\mathbf{P}^{2}}(1)$.

\subsubsection{Le Potier curve}

Define $v\left(E_{\left(\frac{p}{2^{m}}\right)}\right)=\tilde{v}\left(E_{\left(\frac{p}{2^{m}}\right)}\right) / \operatorname{ch}_{0}\left(E_{\left(\frac{p}{2^{m}}\right)}\right)$. We use Chern characters [ $\left.\operatorname{ch}_{0}, \mathrm{ch}_{1}, \mathrm{ch}_{2}\right]$ for the coordinate of $\mathrm{K}_{\mathbb{R}}\left(\mathbf{P}^{2}\right)$. Consider the real projective space $\mathrm{P}\left(\mathrm{K}_{\mathbb{R}}\left(\mathbf{P}^{2}\right)\right)$ with homogeneous coordinate $\left[\mathrm{ch}_{0}, \mathrm{ch}_{1}, \mathrm{ch}_{2}\right]$. We view the locus $\mathrm{ch}_{0}=0$ as the line at infinity, and call $\mathrm{P}\left(\mathrm{K}_{\mathbb{R}}\left(\mathbf{P}^{2}\right)\right)$ the projective $\left\{1, \frac{\mathrm{ch}_{1}}{\mathrm{ch}_{0}}, \frac{\mathrm{ch}_{2}}{\mathrm{ch}_{0}}\right\}$ - plane. The complement of the line at infinity forms an affine real plane, which is referred to as the $\left\{1, \frac{\mathrm{ch}_{1}}{\mathrm{ch}_{0}}, \frac{\mathrm{ch}_{2}}{\mathrm{ch}_{0}}\right\}$-plane. We will define the Le Potier curve on this $\left\{1, \frac{\mathrm{ch}_{1}}{\mathrm{ch}_{0}}, \frac{\mathrm{ch}_{2}}{\mathrm{ch}_{0}}\right\}$-plane.

Let $e\left(\frac{p}{2^{m}}\right)$ be the point on the $\left\{1, \frac{\mathrm{ch}_{1}}{\mathrm{ch}_{0}}, \frac{\mathrm{ch}_{2}}{\mathrm{ch}_{0}}\right\}$-plane with coordinate $v\left(E_{\left(\frac{p}{2^{m}}\right)}\right)$. We associate three more points $e^{+}\left(\frac{p}{2^{m}}\right), e^{l}\left(\frac{p}{2^{m}}\right)$ and $e^{r}\left(\frac{p}{2^{m}}\right)$ to $E_{\left(\frac{p}{2^{m}}\right)}$ on the $\left\{1, \frac{\mathrm{ch}_{1}}{\mathrm{ch}_{0}}, \frac{\mathrm{ch}_{2}}{\mathrm{ch}_{0}}\right\}$-plane. The coordinate of $e^{+}\left(\frac{p}{2^{m}}\right)$ is given as: 


$$
e^{+}\left(\frac{p}{2^{m}}\right):=e\left(\frac{p}{2^{m}}\right)-\left(0,0, \frac{1}{\left(\operatorname{ch}_{0}\left(E_{\left(\frac{p}{2^{m}}\right)}\right)\right)^{2}}\right)
$$

For any real number $a$, let $\Delta_{a}$ be the parabola:

$$
\frac{1}{2}\left(\frac{\mathrm{ch}_{1}}{\mathrm{ch}_{0}}\right)^{2}-\frac{\mathrm{ch}_{2}}{\operatorname{ch}_{0}}=a
$$

on the $\left\{1, \frac{\mathrm{ch}_{1}}{\mathrm{ch}_{0}}, \frac{\mathrm{ch}_{2}}{\mathrm{ch}_{0}}\right\}$-plane. Let $\Delta_{>a}\left(\Delta_{<a}\right)$ be the region $\left\{\left(1, \frac{\mathrm{ch}_{1}}{\mathrm{ch}_{0}}, \frac{\mathrm{ch}_{2}}{\mathrm{ch}_{0}}\right) \mid \frac{1}{2}\left(\frac{\mathrm{ch}_{1}}{\mathrm{ch}_{0}}\right)^{2}-\right.$ $\left.\frac{\mathrm{ch}_{2}}{\operatorname{ch}_{0}}>a(<a)\right\}$. The point $e^{l}\left(\frac{p}{2^{m}}\right)$ is defined to be the intersection of $\Delta_{\frac{1}{2}}$ and the segment $l_{e^{+}\left(\frac{p}{2^{m}}\right) e\left(\frac{p-1}{2^{m}}\right)} ; e^{r}\left(\frac{p}{2^{m}}\right)$ is defined to be the intersection of $\Delta_{\frac{1}{2}}$ and the segment $l_{e^{+}\left(\frac{p}{2^{m}}\right) e\left(\frac{p+1}{2^{m}}\right)}$.

Definition 1.4 (Le Potier Curve) In the $\left\{1, \frac{\mathrm{ch}_{1}}{\mathrm{ch}_{0}}, \frac{\mathrm{ch}_{2}}{\mathrm{ch}_{0}}\right\}$-plane, consider the open region below all the line segments $l_{e^{+}\left(\frac{p}{2^{m}}\right) e^{l}\left(\frac{p}{2^{m}}\right)}, l_{e^{r}\left(\frac{p}{2^{m}}\right) e^{+}\left(\frac{p}{2^{m}}\right)}$ and the curve $\Delta_{\frac{1}{2}}$. The boundary of this open region is a fractal curve in the region between $\Delta_{\frac{1}{2}}$ and $\Delta_{1}$ consisting of line segments $l_{e^{+}\left(\frac{p}{2^{m}}\right) e^{l}\left(\frac{p}{2^{m}}\right)}, l_{e^{r}\left(\frac{p}{2^{m}}\right) e^{+}\left(\frac{p}{2^{m}}\right)}$ for all dyadic numbers $\frac{p}{2^{m}}$ and fractal pieces of points on $\Delta_{\frac{1}{2}}$. We call this curve the Le Potier curve on the $\left\{1, \frac{\mathrm{ch}_{1}}{\mathrm{ch}_{0}}, \frac{\mathrm{ch}_{2}}{\mathrm{ch}_{0}}\right\}$-plane, and denote it by $C_{L P}$. We call the cone in $\mathrm{K}_{\mathbb{R}}\left(\mathbf{P}^{2}\right)$ spanned by the origin and $C_{L P}$ as the Le Potier cone.

We also make a notation for the following open region above $C_{L P}$.

$$
\operatorname{Geo}_{L P}:=\left\{(1, a, b) \mid(1, a, b) \text { is above } C_{L P} \text { and not on any segment } l_{e e^{+}}\right\} \text {. }
$$

Theorem 1.5 (Drezet, Le Potier) There exists a Gieseker semistable coherent sheaf with character $\left(\mathrm{ch}_{0}(>0), \mathrm{ch}_{1}, \mathrm{ch}_{2}\right) \in \mathrm{K}\left(\mathbf{P}^{2}\right)$ if and only if either:

1. it is proportional to an exceptional character $e\left(\frac{p}{2^{m}}\right)$;

2. The point $\left(1, \frac{\mathrm{ch}_{1}}{\mathrm{ch}_{0}}, \frac{\mathrm{ch}_{2}}{\mathrm{ch}_{0}}\right)$ is on or below $C_{L P}$ in the $\left\{1, \frac{\mathrm{ch}_{1}}{\mathrm{ch}_{0}}, \frac{\mathrm{ch}_{2}}{\mathrm{ch}_{0}}\right\}-$ plane.

Remark 1.6 In this article, when we talk about the $\left\{1, \frac{\mathrm{ch}_{1}}{\mathrm{ch}_{0}}, \frac{\mathrm{ch}_{2}}{\mathrm{ch}_{0}}\right\}$-plane, we always assume the $\frac{\mathrm{ch}_{1}}{\mathrm{ch}_{0}}$-axis is horizontal and the $\frac{\mathrm{ch}_{2}}{\mathrm{ch}_{0}}$-axis is vertical. The phrase 'above' is translated as ' $\frac{\mathrm{ch}_{2}}{\mathrm{ch}_{0}}$ coordinates is greater than'. Other words such as: below, right, left can be translated in a similar way.

The full strong exceptional collections on $\mathrm{D}^{\mathrm{b}}\left(\mathbf{P}^{2}\right)$ have been classified by Gorodentsev and Rudakov [15]. In particular, up to a cohomological shift, the collection consists of exceptional bundles on $\mathbf{P}^{2}$. In terms of dyadic numbers, their labels are of three cases: 


$$
\left\{\frac{p-1}{2^{m}}, \frac{p}{2^{m}}, \frac{p+1}{2^{m}}\right\} ;\left\{\frac{p}{2^{m}}, \frac{p+1}{2^{m}}, \frac{p-1}{2^{m}}+3\right\} ;\left\{\frac{p+1}{2^{m}}-3, \frac{p-1}{2^{m}}, \frac{p}{2^{m}}\right\} \text {. }
$$

\subsection{Review: geometric stability conditions}

We briefly recall the definition of stability conditions on a triangulated category from [8]. Let $\mathcal{T}$ be the bounded derived category of coherent sheaves on a smooth projective variety. A pre-stability condition $\sigma=(\mathcal{P}, Z)$ on $\mathcal{T}$ consists of a central charge $Z$ : $\mathrm{K}_{0}(\mathcal{T}) \rightarrow \mathbb{C}$, which is an $\mathbb{R}$-linear homomorphism, and a slicing $\mathcal{P}: \mathbb{R} \rightarrow$ (full additive subcategories of $\mathcal{T}$ ), satisfying the following axioms:

1. For any object $E$ in $\mathcal{P}(\phi)$, we have $Z(E)=m(E) \exp (i \pi \phi)$ for some $m(E) \in$ $\mathbb{R}_{>0}$;

2. $\mathcal{P}(\phi+1)=\mathcal{P}(\phi)[1]$

3. when $\phi_{1}>\phi_{2}$ and $A_{i} \in \operatorname{obj}\left(\mathcal{P}\left(\phi_{i}\right)\right)$, we have $\operatorname{Hom}_{\mathcal{T}}\left(A_{1}, A_{2}\right)=0$;

4. (Harder-Narasimhan filtration) For any object $E$ in $\mathcal{T}$, there is a sequence of real numbers $\phi_{1}>\cdots>\phi_{n}$ and a collection of vanishing triangles $E_{j-1} \rightarrow E_{j} \rightarrow A_{j}$ with $E_{0}=0, E_{n}=E$ and $A_{j} \in \operatorname{obj} \mathcal{P}\left(\phi_{j}\right)$ for all $j$.

A pre-stability condition is called a stability condition if it satisfies the support condition: there exists a quadratic form $Q$ on the vector space $\mathrm{K}_{\mathbb{R}}(\mathcal{T})$ such that

- For any $E \in \operatorname{obj}(\mathcal{P}(\phi)), Q(E) \geq 0$;

- $\left.Q\right|_{\text {Ker } Z}$ is negative definite.

For the rest part of this section, we will follow the line of [9] and [4] and conclude that the space of geometric stability condition on $\mathbf{P}^{2}$ is a $\tilde{G L}^{+}(2, \mathbb{R})$ fiber space over $\mathrm{Geo}_{L P}$.

Definition 1.7 A stability condition $\sigma$ on $\mathrm{D}^{\mathrm{b}}\left(\mathbf{P}^{2}\right)$ is called geometric if all skyscraper sheaves $k(x)$ are $\sigma$-stable with the same phase. We denote the subset of all geometric stability conditions by $\operatorname{Stab}^{\mathrm{Geo}}\left(\mathbf{P}^{2}\right)$.

Let $s$ be a real number, a torsion pair of coherent sheaves on $\mathbf{P}^{2}$ is given by:

$\mathrm{Coh}_{\leq s}$ : the subcategory of $\operatorname{Coh}\left(\mathbf{P}^{2}\right)$ generated by slope semistable sheaves of slope $\leq s$ by extension.

$\mathrm{Coh}_{>s}$ : the subcategory of $\operatorname{Coh}\left(\mathbf{P}^{2}\right)$ generated by slope semistable sheaves of slope $>s$ and torsion sheaves by extension.

$\mathrm{Coh}_{\# s}:=\left\langle\mathrm{Coh}_{\leq s}[1], \mathrm{Coh}_{>s}\right\rangle$

Definition 1.8 Given $(s, q) \in \mathrm{Geo}_{L P}$, the $\sigma_{s, q}=\left(Z_{s, q}, \mathcal{P}_{s, q}\right)$ on $\mathrm{D}^{\mathrm{b}}\left(\mathbf{P}^{2}\right)$ is defined by the central charge $Z_{s, q}$ on the heart $\mathcal{P}_{s, q}((0,1])=\mathrm{Coh}_{\# s}$.

$$
Z_{s, q}(E):=\left(-\operatorname{ch}_{2}(E)+q \cdot \operatorname{ch}_{0}(E)\right)+i\left(\operatorname{ch}_{1}(E)-s \cdot \operatorname{ch}_{0}(E)\right) .
$$

Let the phase function $\phi_{s, q}$ be defined for objects in $\mathrm{Coh}_{\# s}: \phi_{s, q}(E):=(1 / \pi) \arg \left(Z_{s, q}\right.$ $(E))$. For $\phi \in(0,1]$, each slice $\mathcal{P}(\phi)$ is formed by the semistable objects (with respect to $Z_{s, q}$ ) with phase $\phi_{s, q}=\phi$. 
Table 1 List of notations

\begin{tabular}{|c|c|}
\hline $\mathcal{H}_{P}$ & The right half plane with $\frac{\mathrm{ch}_{1}}{\mathrm{ch}_{0}}>s$, or $\frac{\mathrm{ch}_{1}}{\mathrm{ch}_{0}}=s$ and $\frac{\mathrm{ch}_{2}}{\mathrm{ch}_{0}}>q$ \\
\hline $\mathcal{H}_{E}$ & $H_{v(E)}$ when $v(E)$ is not at infinity \\
\hline$L_{E F}$ & The line on $\mathrm{P}\left(\mathrm{K}_{\mathbb{R}}\left(\mathbf{P}^{2}\right)\right)$ across $v(E)$ and $v(F)$ \\
\hline$L_{E P}$ & The line on $\mathrm{P}\left(\mathrm{K}_{\mathbb{R}}\left(\mathbf{P}^{2}\right)\right)$ across $v(E)$ and $P$ \\
\hline$l_{E F}\left(l_{E P}\right)$ & The line segment $\overline{v(E) v(F)}(\overline{v(E) P})$ on the $\left\{1, \frac{\mathrm{ch}_{1}}{\mathrm{ch}_{0}}, \frac{\mathrm{ch}_{2}}{\mathrm{ch}_{0}}\right\}$-plane \\
\hline$l_{E F}^{r}$ & The ray along $L_{E F}$ from $v(F)$ to infinity and does not contain $v(E)$ \\
\hline$l_{E F}^{+}$ & The ray along $L_{E F}$ from $v(E)$ on the $\mathcal{H}_{E}$ part \\
\hline$l_{E+}$ & The ray segment on $L_{E(0,0,1)}$ on the $\mathcal{H}_{E}$ part \\
\hline$l_{E-}$ & The ray segment on $L_{E(0,0,-1)}$ outside the $\mathcal{H}_{v(E)}$ part \\
\hline $\mathcal{E}$ & A triple of ordered exceptional objects $\left\{E_{1}, E_{2}, E_{3}\right\}$ \\
\hline $\mathrm{TR}_{\mathcal{E}}$ & The inner points in the triangle bounded by $l_{E_{i} E_{j}}$, for $1 \leq i<j \leq 3$. \\
\hline$e_{i}^{*}$ & $v^{*}\left(E_{i}\right)$ as defined in Sect. $1, *$ can be $+, l, r$ or blank \\
\hline $\mathrm{MZ}_{\mathcal{E}}$ & The inner points of region bounded by $l_{e_{1} e_{1}^{+}}, l_{e_{1}^{+} e_{2}} l_{e_{2} e_{3}^{+}}, l_{e_{3}^{+} e_{3}}$ and $l_{e_{3} e_{1}}$ \\
\hline
\end{tabular}

Remark 1.9 This definition of the central charge $Z_{s, q}$ is slightly different from the usual case as that in the [1]. The imaginary parts are defined in the same way, but the real part is different from the usual case by a scalar times the imaginary part. We would like to use the version here because its kernel is clear. In addition, if we write $P$ for the point $(1, s, q)$, then the phase (times $\pi$ ) of an object $E$ in $\mathrm{Coh}_{\# s}$ is the angle spanned by the rays $l_{P E}^{+}$and $l_{P-}$ (for definition, see Table 1 ) at $P$ on the $\mathcal{H}_{P}$ half plane.

Proposition 1.10 For any $(s, q) \in \mathrm{Geo}_{L P}, \sigma_{s, q}=\left(Z_{s, q}, \mathcal{P}_{s, q}\right)$ is a geometric stability condition.

For the proof, readers are referred to the arguments in [9] and [4] Corollary 4.6, which also work well in the $\mathbf{P}^{2}$ case. $\mathrm{Up}$ to the $\tilde{G L}^{+}(2, \mathbb{R})$-action, geometric stability conditions can only be of the form given in Proposition 1.10 .

Notation 1.11 Given a point $P=(1, s, q)$ in $\mathrm{Geo}_{L P}$, we will also write $\sigma_{P}, \phi_{P}$, $\operatorname{Coh}_{P}\left(\mathbf{P}^{2}\right)$ and $Z_{P}$ for the stability condition $\sigma_{s, q}$, the phase function $\phi_{s, q}$, the tilt heart $\mathrm{Coh}_{\# s}\left(\mathbf{P}^{2}\right)$ and the central charge $Z_{s, q}$ respectively.

Proposition 1.12 ([9] Proposition 10.3, [4] Section 3) Let $\sigma=(Z, \mathcal{P})$ be a geometric stability condition with all skyscraper sheaves $k(x)$ in $\mathcal{P}(1)$. Then the heart $\mathcal{P}((0,1])$ is $\mathrm{Coh}_{\# \text { s }}$ for some real number $s$. The central charge $Z$ can be written in the form of

$$
-\mathrm{ch}_{2}+a \cdot \mathrm{ch}_{1}+b \cdot \mathrm{ch}_{0}
$$

The complex numbers $a$ and $b$ satisfies the following conditions:

- $\Im a>0, \frac{\Im b}{\Im a}=s$; 
- $\left(\frac{\Im b}{\Im a}, \frac{\Re a \Im b}{\Im a}+\Re b\right)$ is in $\mathrm{Geo}_{L P}$.

Knowing the classification result Theorem 1.5 on stable characters, the property is proved in the same way as that in the local $\mathbf{P}^{2}$ and K3 surfaces cases.

\subsection{Destabilizing walls}

We collect some small but useful lemmas in this section.

Definition 1.13 We call a stability condition non-degenerate if the image of its central charge is not contained in a real line. We write $\operatorname{Stab}^{\text {nd }}\left(\mathbf{P}^{2}\right)$ for the set of all nondegenerate stability conditions.

Note that by Proposition 1.12, $\operatorname{Stab}^{\mathrm{Geo}}\left(\mathbf{P}^{2}\right) \subset \operatorname{Stab}^{\text {nd }}\left(\mathbf{P}^{2}\right)$. In this Picard rank 1 case, the kernel map on the central charge is well-defined on $\operatorname{Stab}^{\text {nd }}\left(\mathbf{P}^{2}\right)$.

$$
\text { Ker }: \operatorname{Stab}^{\text {nd }}\left(\mathbf{P}^{2}\right) \rightarrow \mathrm{P}\left(\mathrm{K}_{\mathbb{R}}\left(\mathbf{P}^{2}\right)\right)
$$

Lemma $1.14 \tilde{\mathrm{GL}}^{+}(2, \mathbb{R})$ acts freely on $\mathrm{Stab}^{\mathrm{nd}}\left(\mathbf{P}^{2}\right)$ with closed orbits, and

$$
\operatorname{Ker}: \operatorname{Stab}^{\text {nd }}\left(\mathbf{P}^{2}\right) / \tilde{G L}^{+}(2, \mathbb{R}) \rightarrow \mathrm{P}\left(\mathrm{K}_{\mathbb{R}}\left(\mathbf{P}^{2}\right)\right)
$$

is a local homeomorphism.

Proof By Theorem 1.2 in [8], $\operatorname{Stab}^{\text {nd }}\left(\mathbf{P}^{2}\right) \rightarrow \operatorname{Hom}_{\mathbb{Z}}\left(\mathrm{K}\left(\mathbf{P}^{2}\right), \mathbb{C}\right)$ is a local homeomorphism. The image is in the non-degenerate part of $\operatorname{Hom}_{\mathbb{Z}}\left(\mathrm{K}\left(\mathbf{P}^{2}\right), \mathbb{C}\right) . \operatorname{Hom}_{\mathbb{Z}}^{\text {nd }}\left(\mathrm{K}\left(\mathbf{P}^{2}\right), \mathbb{C}\right)$ $/ \mathrm{GL}^{+}(2, \mathbb{R})$ is just the quotient Grassmannian $\mathrm{Gr}_{2}(3)$ as a topological space.

Corollary $1.15 \tilde{\mathrm{GL}}^{+}(2, \mathbb{R})$ acts freely on $\mathrm{Stab}^{\mathrm{Geo}}\left(\mathbf{P}^{2}\right)$, and

$$
\operatorname{Stab}^{\mathrm{Geo}}\left(\mathbf{P}^{2}\right) / \tilde{\mathrm{GL}}^{+}(2, \mathbb{R}) \simeq \text { GeoLP. }
$$

Lemma 1.16 Let $Z$ be a non-degenerate central charge, $v$ and $w$ be two non-zero characters, then

$$
Z(v) / / Z(w)
$$

if and only if $v, w$ and the line $\operatorname{Ker} Z$ in $\mathrm{K}_{\mathbb{R}}\left(\mathbf{P}^{2}\right)$ span a two-dimensional plane.

Proof $Z(v) / / Z(w)$ if and only if $Z(a v+b w)=0$ for some $a, b \in \mathbb{R} . v, w, a v+b w$ and $O$ are on the same plane.

Lemma 1.17 Let $P$ be a point in $\mathrm{Geo}_{L P}, E$ and $F$ be two objects in $\mathrm{Coh}_{P}$. The phase

$$
\phi_{P}(E)>\phi_{P}(F)
$$

if and only if the ray $l_{P E}^{+}$is above $l_{P F}^{+}$. 
Proof By the definition of $l_{P E}^{+}, l_{P-}, Z_{P}$ and Remark 1.9, the angle spanned by the rays $l_{P E}^{+}$and $l_{P-}$ at point $P$ on the $\left\{1, \frac{\mathrm{ch}_{1}}{\mathrm{ch}_{0}}, \frac{\mathrm{ch}_{2}}{\mathrm{ch}_{0}}\right\}-$ plane is $\pi \phi_{P}(E)$. The statement is clear.

Proposition 1.18 Let $E$ be an $\sigma_{P}$-stable object, then one of the following cases will hold:

1. $\tilde{v}(E)$ is not in the open cone spanned by $\mathrm{Geo}_{L P}$ and the origin.

2. There exists a slope semistable sheaf $F$ such that the point $P$ is in the region bounded by $l_{E F}^{r}$ and $l_{F-}$.

In either case, the line $l_{E P}$ is not inside $\mathrm{Geo}_{L P}$. In particular, at least one of $v(E)$ and $P$ is outside $\Delta_{<0}$.

Proof Suppose $\tilde{v}(E)$ is in the $\mathrm{Geo}_{L P}$-cone, in particular, $\mathrm{ch}_{0}$ is not 0 .

When $\operatorname{ch}_{0}(E)>0, \mathrm{H}^{0}(E)$ is non-zero. Let $F=\mathrm{H}^{0}(E)_{\text {min }}$ be the quotient sheaf of $\mathrm{H}^{0}(E)$ with the minimum slope. Let $D$ be $\mathrm{H}^{-1}(E)$ and $G$ be the kernel of $\mathrm{H}^{0}(E) \rightarrow F$. We have $\mu(D)<\mu(F)<\mu(G)$, when $D$ and $G$ are non-zero. We have the relation

$$
\frac{\operatorname{ch}_{1}(E)}{\operatorname{ch}_{0}(E)}=\frac{\operatorname{ch}_{1}(F)+\operatorname{ch}_{1}(G)-\operatorname{ch}_{1}(D)}{\operatorname{ch}_{0}(F)+\operatorname{ch}_{0}(G)-\operatorname{ch}_{0}(D)} \geq \frac{\operatorname{ch}_{1}(F)}{\operatorname{ch}_{0}(F)}
$$

The equality only holds when $D$ and $G$ are both zero, but this is not possible as else $v(E)$ is equal to $v(F)$, which is inside $\operatorname{Geo}_{L P}$ by Theorem 1.5. Therefore, $v(F)$ is to the left of $v(E)$ on the $\left\{1, \frac{\mathrm{ch}_{1}}{\mathrm{ch}_{0}}, \frac{\mathrm{ch}_{2}}{\mathrm{ch}_{0}}\right\}$-plane. Let $P=(1, s, q)$, as $F \in \mathrm{Coh}_{>s}, P$ is to the left of $v(F)$. In addition, as $\phi_{P}(E)<\phi_{P}(F)$, by Lemma 1.17, $P$ is below the line $L_{E F}$. Therefore, $P$ is in the region bounded by $l_{E F}^{r}$ and $l_{F-}$.

When $\operatorname{ch}_{0}(E)<0$, let $F=\mathrm{H}^{-1}(E)_{\text {max }}$ be the subsheaf of $\mathrm{H}^{-1}(E)$ with maximum Mumford slope. By the same argument, $v(F)$ is to the right of $v(E)$. As $F \in \mathrm{Coh}_{\leq s}$, $P$ is to the right of $v(F)$ or on the line $L_{F(0,0,1)}$. In addition, as $\phi_{P}(F[1])<\phi_{P}(E)$, by Lemma 1.17, $P$ is below $L_{E F}$. As $l_{F-}$ does not intersect $\mathrm{Geo}_{L P}, P$ is not on $L_{F(0,0,1)}$. Therefore, $P$ is in the region bounded by $l_{E F}^{r}$ and $l_{F-}$.

For the last statement, the region $\Delta_{<0}$ is bounded by a parabola and is convex. For any $v(E)$ and $P$ that are both in the region, $l_{E P}$ is also in the region which is contained in $\mathrm{Geo}_{L P}$.

Corollary 1.19 Let $E$ be an exceptional bundle, and $P=(1, s, q)$ be a point in $\mathrm{Geo}_{L P}$, then $E$ is $\sigma_{P}$-stable if $s<\mu(E)$ and $l_{E P}$ is contained in $\mathrm{Geo}_{L P}$. On the shifted side, $E[1]$ is $\sigma_{P}$-stable, if $\mu(E) \leq s$ and $l_{E P}$ is contained in $\mathrm{Geo}_{L P}$.

Proof Assume $s<\mu(E)$ and $E$ is not $\sigma_{P}$-stable, then there is a $\sigma_{P}$-stable object $F$ destabilizing $E$. By the exact sequence:

$0 \rightarrow \mathrm{H}^{-1}(F) \rightarrow \mathrm{H}^{-1}(E) \rightarrow \mathrm{H}^{-1}(E / F) \rightarrow \mathrm{H}^{0}(F) \rightarrow \mathrm{H}^{0}(E) \rightarrow \mathrm{H}^{0}(E / F) \rightarrow 0$

we get $\mathrm{H}^{-1}(F) \subset \mathrm{H}^{-1}(E)=0$, and $v(F)$ is between $L_{P(0,0,1)}$ and $L_{E(0,0,1)}$. As $\phi_{P}(F) \geq \phi_{P}(E)$ by assumption, by Lemma $1.17, v(F)$ is in the region bounded by $l_{P+}, l_{P E}$, and $l_{E+}$. As $l_{E P}$ is in $\mathrm{Geo}_{L P}$, the whole open region bounded by these three 
segments is also in $\mathrm{Geo}_{L P}$. The whole line segment $l_{F P}$ is contained in $\mathrm{Geo}_{L P}$ (unless $v(F)=v(E)$, which implies $E=F)$. By Proposition $1.18, F$ is not $\sigma_{P}$-stable, which is a contradiction. The $s \geq \mu(E)$ case is proved in a similar way.

Remark 1.20 The condition ' $l_{E P}$ is contained in $\mathrm{Geo}_{L P}$ ' is also a necessary condition. Any ray from $v(E)$ only intersects the Le Potier curve once, and only intersects finitely many $e e^{+}$segments. Assume we are in the $s<\mu(E)$ case and $l_{E P}$ intersects some $e e^{+}$ segments, we may choose the one (denoted by $F$ ) with minimum $\frac{\mathrm{ch}_{1}}{\mathrm{ch}_{0}}$ coordinate. The segment $l_{F P}$ is contained in $\mathrm{Geo}_{L P}$, and the $\phi_{s, q}(F)>\phi_{s, q}(E)$. By [15], $\operatorname{Hom}(F, E)$ $\neq 0$ when $\mu(F)<\mu(E)$. This leads to a contradiction if $E$ is $\sigma_{s, q}$-stable.

\section{Algebraic stability conditions}

\subsection{Review: algebraic stability conditions}

Definition 2.1 We call an ordered set $\mathcal{E}=\left\{E_{1}, E_{2}, E_{3}\right\}$ exceptional triple on $\mathrm{D}^{\mathrm{b}}\left(\mathbf{P}^{2}\right)$ if $\mathcal{E}$ is a full strong exceptional collection of coherent sheaves on $\mathrm{D}^{\mathrm{b}}\left(\mathbf{P}^{2}\right)$.

We will write $e_{i}^{*}$ for $e^{*}\left(E_{i}\right)$ as the associated points on the $\left\{1, \frac{\mathrm{ch}_{1}}{\mathrm{ch}_{0}}, \frac{\mathrm{ch}_{2}}{\mathrm{ch}}\right\}$-plane, where $i=1,2,3$ and $*$ could be,$+ l$, or $r$. By the definition of $e^{*}$ 's, the relation of dyadic numbers (\$), and Serre duality, the points $e_{1}^{+}, e_{1}^{r}, e_{2}, e_{3}$ are collinear on the line of $\chi\left(-, E_{1}\right)=0$, and $e_{3}^{+}, e_{3}^{l}, e_{2}, e_{1}$ are collinear on the line of $\chi\left(E_{3},-\right)=0$.

We are now ready to recall the construction of algebraic stability conditions with respect to exceptional triples.

Proposition 2.2 ([20] Section 3) Let $\mathcal{E}$ be an exceptional triple on $\mathrm{D}^{\mathrm{b}}\left(\mathbf{P}^{2}\right)$, for any positive real numbers $m_{1}, m_{2}, m_{3}$ and real numbers $\phi_{1}, \phi_{2}, \phi_{3}$ such that:

$$
\phi_{1}<\phi_{2}<\phi_{3} \text {, and } \phi_{1}+1<\phi_{3} \text {. }
$$

There is a unique stability condition $\sigma=(Z, \mathcal{P})$ such that

1. each $E_{j}$ is stable with phase $\phi_{j}$;

2. $Z\left(E_{j}\right)=m_{j} e^{i \pi \phi_{j}}$.

Definition 2.3 Given an exceptional triple $\mathcal{E}=\left\{E_{1}, E_{2}, E_{3}\right\}$ on $\mathrm{D}^{\mathrm{b}}\left(\mathbf{P}^{2}\right)$, we write $\Theta_{\mathcal{E}}$ as the space of all stability conditions in Proposition 2.2. $\Theta_{\mathcal{E}}$ is parametrized by

$$
\left\{\left(m_{1}, m_{2}, m_{3}, \phi_{1}, \phi_{2}, \phi_{3}\right) \in\left(\mathbb{R}_{>0}\right)^{3} \times \mathbb{R}^{3} \mid \phi_{1}<\phi_{2}<\phi_{3}, \phi_{1}+1<\phi_{3}\right\} .
$$

We make the following notations for some subsets of $\Theta_{\mathcal{E}}$.

- $\Theta_{\mathcal{E}}^{\nabla}:=\left\{\sigma \in \Theta_{\mathcal{E}} \mid \phi_{2}-\phi_{1} \leq 1, \phi_{3}-\phi_{2} \leq 1\right.$ and $\left.\phi_{3}-\phi_{1} \neq 2\right\}$;

- $\Theta_{\mathcal{E}}^{\mathrm{Geo}}:=\Theta_{\mathcal{E}} \cap \mathrm{Stab}^{\mathrm{Geo}}$

- $\Theta_{\mathcal{E}}^{\text {Pure }}:=\left\{\sigma \in \Theta_{\mathcal{E}} \mid \phi_{2}-\phi_{1} \geq 1\right.$ and $\left.\phi_{3}-\phi_{2} \geq 1\right\}$;

- $\Theta_{\mathcal{E}, E_{1}}^{+}:=\left\{\sigma \in \Theta_{\mathcal{E}} \mid \phi_{3}-\phi_{2}<1\right\} \backslash \Theta_{\mathcal{E}}^{\mathrm{Geo}}$;

- $\Theta_{\mathcal{E}, E_{3}}^{-}:=\left\{\sigma \in \Theta_{\mathcal{E}} \mid \phi_{2}-\phi_{1}<1\right\} \backslash \Theta_{\mathcal{E}}^{\mathrm{Geo}}$. 


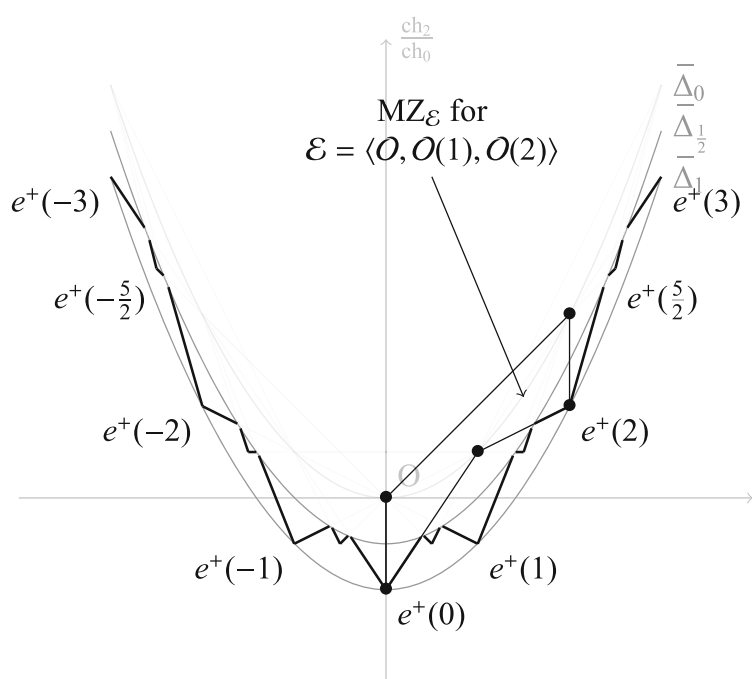

Fig. 1 The Le Potier curve $C_{L P}$

We denote $\mathrm{Stab}^{\mathrm{Alg}}$ as the union of $\Theta_{\mathcal{E}}$ for all exceptional triples on $\mathrm{D}^{\mathrm{b}}\left(\mathbf{P}^{2}\right)$, and call the element of it the algebraic stability conditions.

Lemma 2.4 Let $\mathcal{E}=\left\{E_{1}, E_{2}, E_{3}\right\}$ be an exceptional triple, and $\sigma$ be a stability condition in $\Theta_{\mathcal{E}}^{\text {Pure }}$. The only $\sigma$-stable objects are $E_{i}[n]$ for $i=1,2,3$ and $n \in \mathbb{Z}$.

Proof Let $F$ be a $\sigma$-stable object, we may assume it is in the heart $\left\langle E_{1}[a], E_{2}[b], E_{3}\right\rangle$, where $a, b \in \mathbb{Z}$ such that $b \geq 1$ and $a-b \geq 1$. When $b \geq 2$, as $\operatorname{Ext}^{1}\left(E_{3},-\right)$ and $\operatorname{Ext}^{1}\left(-, E_{3}\right)$ are 0 for any other generators, $F$ is either $E_{3}$ or belongs to $\left\langle E_{1}[a], E_{2}[b]\right\rangle$. If $a-b \geq 2$, then $F$ is either $E_{1}[a]$, or $E_{2}[b]$. Else we have $a=b+1$, and we have the sequence $E_{2}^{\oplus n_{2}}[b] \rightarrow F \rightarrow E_{1}^{\oplus n_{2}}[b+1]$ in the heart. As $\phi\left(E_{2}[b]\right) \geq \phi\left(E_{1}[b+1]\right)$, $F$ is not $\sigma$-stable unless $F$ is $E_{2}[b]$ or $E_{1}[a]$.

We may therefore assume $a=2$ and $b=1$, then $F$ is in the form of $E_{1}^{\oplus n_{1}} \rightarrow$ $E_{2}^{\oplus n_{2}} \rightarrow E_{3}^{\oplus n_{3}}$. As $\phi_{3} \geq \phi_{2}+1 \geq \phi_{1}+2, F$ is either $E_{3}, E_{2}$ [1] or $E_{1}$ [2].

\subsection{Common areas of geometric and algebraic stability conditions}

Let $\mathcal{E}=\left\{E_{1}, E_{2}, E_{3}\right\}$ be an exceptional triple, in this section, we will explain how the algebraic part $\Theta_{\mathcal{E}}$ 'glues' on to $\mathrm{Stab}^{\mathrm{Geo}}$. We denote $\mathrm{TR}_{\mathcal{E}}$ the triangle region on $\left\{1, \frac{\mathrm{ch}_{1}}{\mathrm{ch}_{0}}, \frac{\mathrm{ch}_{2}}{\mathrm{ch}_{0}}\right\}$-plane bounded anticlockwise by line segments $l_{e_{1} e_{2}}, l_{e_{2} e_{3}}$ and $l_{e_{3} e_{1}}$ (the edges $l_{e_{1} e_{2}}, l_{e_{2} e_{3}}$ are defined to be in the $\mathrm{TR}_{\mathcal{E}}$, the three vertices are not). We denote $\mathrm{MZ}_{\mathcal{E}}$ as the open region on $\left\{1, \frac{\mathrm{ch}_{1}}{\mathrm{ch}_{0}}, \frac{\mathrm{ch}_{2}}{\mathrm{ch}_{0}}\right\}$-plane bounded anticlockwise by line segments $l_{e_{1} e_{1}^{+}}, l_{e_{1}^{+} e_{2}}, l_{e_{2} e_{3}^{+}}, l_{e_{3}^{+} e_{3}}$ and $l_{e_{3} e_{1}}$. A picture of $\mathrm{MZ}_{\mathcal{E}}$ for $\mathcal{E}=\langle\mathcal{O}, \mathcal{O}(1), \mathcal{O}(2)\rangle$ is drawn in Fig. 1.

Proposition 2.5 Let $\mathcal{E}$ be an exceptional triple, then we have: 
1. $\Theta_{\mathcal{E}}^{\nabla}=\tilde{G L}^{+}(2, \mathbb{R}) \cdot\left\{\sigma_{s, q} \in \operatorname{Stab}^{\mathrm{Geo}}\left(\mathbf{P}^{2}\right) \mid(1, s, q) \in \mathrm{TR}_{\mathcal{E}}\right\}$. In particular, $\Theta_{\mathcal{E}}^{\nabla}$ is in $\Theta_{\mathcal{E}}^{\mathrm{Geo}}$.

2. $\Theta_{\mathcal{E}}^{\mathrm{Geo}}=\tilde{\mathrm{GL}}^{+}(2, \mathbb{R}) \cdot\left\{\sigma_{s, q} \in \operatorname{Stab}^{\mathrm{Geo}}\left(\mathbf{P}^{2}\right) \mid(1, s, q) \in \mathrm{MZ}_{\mathcal{E}}\right\}$.

Proof We first prove the second statement. As $\mathrm{MZ}_{\mathcal{E}}$ is contained in $\mathrm{Geo}_{L P}$, by Corollary $1.19, E_{2}$ is $\sigma_{s, q}$-stable for any point $(1, s, q)$ in $\mathrm{MZ}_{\mathcal{E}}$. As $e_{1}^{+}, e_{1}^{r}, e_{2}, e_{3}$ are collinear on the line of $\chi\left(-, E_{1}\right)=0$, for any point $P$ in $\mathrm{MZ}_{\mathcal{E}}, l_{E P}$ is contained in $\mathrm{Geo}_{L P}$. By Corollary $1.19, E_{3}$ is stable in $\mathrm{MZ}_{\mathcal{E}}$. For the same reason, $E_{1}$ is stable $\mathrm{MZ}_{\mathcal{E}}$.

For any $(1, s, q)$ in $\mathrm{MZ}_{\mathcal{E}}, E_{3}$ and $E_{1}[1]$ are in the heart $\mathrm{Coh}_{\# s}$. By Lemma 1.17, $\phi_{s, q}\left(E_{1}[1]\right)<\phi_{s, q}\left(E_{3}\right)$, hence

$$
\phi_{s, q}\left(E_{3}\right)-\phi_{s, q}\left(E_{1}\right)>1
$$

When $s \geq \mu\left(E_{2}\right), E_{3}$ and $E_{2}[1]$ are in the heart $\mathrm{Coh}_{\# s}$, we have

$$
\phi_{s, q}\left(E_{3}\right)-\phi_{s, q}\left(E_{2}\right)>0
$$

As $(1, s, q)$ is above $l_{e_{1} e_{2}}$, by Lemma 1.17 , we also have

$$
\phi_{s, q}\left(E_{2}\right)-\phi_{s, q}\left(E_{1}\right)>0
$$

When $s<\mu\left(E_{2}\right)$, by a similar argument we also have the same inequalities for $\phi_{s, q}\left(E_{i}\right)$ 's. By Proposition 2.2, we get the embedding

$$
\operatorname{Ker}^{-1}\left(\mathrm{MZ}_{\mathcal{E}}\right) \cap \operatorname{Stab}^{\mathrm{Geo}} \hookrightarrow \Theta_{\mathcal{E}} \cap \operatorname{Stab}^{n d} \stackrel{\mathrm{Ker}}{\longrightarrow} \mathrm{P}\left(\mathrm{K}_{\mathbb{R}}\left(\mathbf{P}^{2}\right)\right)
$$

For $(1, s, q)$ outside the area $\mathrm{MZ}_{\mathcal{E}}$, we have either $\phi_{s, q}\left(E_{3}\right)-\phi_{s, q}\left(E_{1}\right) \leq 1, \phi_{s, q}\left(E_{2}\right)$ $\leq \phi_{s, q}\left(E_{1}\right)$, or $\phi_{s, q}\left(E_{3}\right) \leq \phi_{s, q}\left(E_{2}\right)$. Because either $E_{1}$ and $E_{3}$ are in the same heart (when $s>\mu\left(E_{3}\right)$ or $s \leq \mu\left(E_{1}\right)$ ), or the slope $\phi_{s, q}\left(E_{1}[1]\right)$ is greater than $\phi_{s, q}\left(E_{3}\right)$; or both $E_{1}[1]$ and $E_{2}[1]$ are in $\operatorname{Coh}_{\# s}$ but $(1, s, q)$ is below $l_{e_{1} e_{2}}$; or both $E_{2}$ and $E_{3}$ are in $\mathrm{Coh}_{\# s}$ but $(1, s, q)$ is below $l_{e_{2} e_{3}}$. Hence $\sigma_{s, q}$ is not contained in $\Theta_{\mathcal{E}}$, this finishes the second statement of the proposition.

For the first statement, since $\phi_{3}-\phi_{1}$ is not an integer, $\Theta_{\mathcal{E}}^{\nabla} \in \mathrm{Stab}^{\text {nd }}$. The image of $\operatorname{Ker}\left(\Theta_{\mathcal{E}}^{\nabla}\right)$ is in $\operatorname{Tr}_{\mathcal{E}}$. By the previous argument, we also have the embedding

$$
\left(\operatorname{Ker}^{-1}\left(\mathrm{TR}_{\mathcal{E}}\right) \cap \operatorname{Stab}^{\mathrm{Geo}}\right) / \tilde{\mathrm{GL}}^{+}(2, \mathbb{R}) \hookrightarrow \Theta_{\mathcal{E}}^{\nabla} / \tilde{G L}^{+}(2, \mathbb{R}) \stackrel{\mathrm{Ker}}{\longrightarrow} \operatorname{TR}_{\mathcal{E}} \subset \mathrm{P}\left(\mathrm{K}_{\mathbb{R}}\left(\mathbf{P}^{2}\right)\right)
$$

The map Ker is local homeomorphism and the composition is an isomorphism. Since $\Theta_{\mathcal{E}}^{\nabla}$ is path connected, the two maps are both isomorphism. We get the first statement of the proposition. 


\section{Cell decomposition and contractibility}

\subsection{Neighbor cells of geometric stability conditions}

Proposition and Definition 3.1 (Definition of $\Theta_{E}^{ \pm}$) Given exceptional triples $\mathcal{E}$ and $\mathcal{E}^{\prime}$ on $\mathrm{D}^{\mathrm{b}}\left(\mathbf{P}^{2}\right)$ with the same $E_{1}=E_{1}^{\prime}=E$, then $\Theta_{\mathcal{E}, E_{1}}^{+}=\Theta_{\mathcal{E}^{\prime}, E_{1}^{\prime}}^{+}$. We denote this subspace by $\Theta_{E}^{+}$. In a similar way, we can define a subspace $\Theta_{E}^{-}$.

Proof Let the three objects in $\mathcal{E}\left(\mathcal{E}^{\prime}\right)$ be $E, E_{2}, E_{3}\left(E, E_{2}^{\prime}, E_{3}^{\prime}\right)$. By [15], $E_{2}^{\prime}, E_{3}^{\prime}$ is constructed from $E_{2}, E_{3}$ by consecutive left or right mutations. Without loss of generality, we may assume $\left(E_{2}^{\prime}, E_{3}^{\prime}\right)$ is just $\left(L_{E_{2}} E_{3}, E_{2}\right)$.

By [20] Proposition 3.17, at a point $(\vec{m}, \vec{\phi})$ in $\Theta_{\mathcal{E}}$, when $\phi_{3}<\phi_{2}+1, L_{E_{2}} E_{3}$ is stable at the point and its phase satisfies

$$
\phi_{3}-1<\phi\left(L_{E_{2}} E_{3}\right)<\phi_{2} \text {. }
$$

When $\phi_{2}-\phi_{1}>1$, this is a stability condition in $\Theta_{\mathcal{E}^{\prime}}$. Under the coordinate of $\Theta_{\mathcal{E}^{\prime}}$, $\phi_{3}^{\prime}-\phi_{2}^{\prime}=\phi_{2}-\phi\left(L_{E_{2}} E_{3}\right)<1$. Note that $\Theta_{\mathcal{E}}\left(\phi_{2}-\phi_{1} \leq 1, \phi_{3}-\phi_{2}<1\right) \subset \operatorname{Stab}^{\mathrm{Geo}}$, we have $\Theta_{\mathcal{E}, E}^{+} \subset \Theta_{\mathcal{E}^{\prime}, E}$.

On the other hand, at a point $\left(\overrightarrow{m^{\prime}}, \overrightarrow{\phi^{\prime}}\right)$ in $\Theta_{\mathcal{E}^{\prime}}$, when $\phi_{3}^{\prime}<\phi_{2}^{\prime}+1, R_{E_{3}^{\prime}} E_{2}^{\prime}\left(=E_{3}\right)$ is stable at the point and

$$
\phi_{3}^{\prime}<\phi\left(E_{3}\right)<\phi_{2}^{\prime}+1
$$

Under the coordinate of $\Theta_{\mathcal{E}}, \phi_{3}-\phi_{2}<\phi_{2}^{\prime}+1-\phi_{3}^{\prime}<1$. Hence $\Theta_{\mathcal{E}^{\prime}, E}^{+} \subset \Theta_{\mathcal{E}, E}$.

Remark $3.2 \Theta_{E}^{-}$is a chamber that the skyscraper sheaf $k(x)$ is destabilized by $E$. $\Theta_{E}^{+}$ is a chamber in which the skyscraper sheaf $k(x)$ is co-destabilized by $E$ [1]. We may also use the notation $\Theta_{\mathcal{E}, E_{1}}^{+}$in some situations, since it has the chart induced from $\Theta_{\mathcal{E}}$.

\subsection{Boundary of geometric stability conditions}

Lemma 3.3 Let $E$ and $F$ be two exceptional bundles such that $\mu(E)<\mu(F)$, then $E$ is not stable under any stability condition in $\Theta_{F}^{+}$and $F$ is not stable under any stability condition in $\Theta_{E}^{-}$.

Proof Let $\mathcal{E}=\left\{E_{1}, E_{2}, E_{3}=E\right\}$ be an exceptional triple extended from $E$. We may choose $E_{2}$ such that $\mu\left(E_{2}\right)<\mu(F)-3$. This can be done because of the correspondence between dyadic triples (\$) and exceptional triples. In particular, we may choose dyadic triples (\$) of the second type for some $q$ large enough.

By [15], since $\mu(E)<\mu(F), \operatorname{Hom}(E, F) \neq 0$. Therefore, we have $\phi(F)>\phi(E)$. On the other hand, $\operatorname{Hom}\left(F, E_{1}[2]\right)=\operatorname{Hom}\left(E_{1}, F(-3)\right) \neq 0$, as by the choice of $\mathcal{E}$, $\mu\left(E_{1}\right)<\mu(F(-3))$. Therefore, $\phi(F)<\phi\left(E_{1}[2]\right)$.

Now we have $\phi\left(E_{3}\right)<\phi(F)<\phi\left(E_{1}[2]\right)$, this implies $\phi_{3}-\phi_{1}<2$. The point $(\vec{m}, \vec{\phi})$ is in the region $\Theta_{\mathcal{E}}\left(\phi_{3}-\phi_{1}<2, \phi_{2}-\phi_{1}<1\right)$. Since $\Theta_{\mathcal{E}}^{\nabla} \subset$ Stab $^{\text {Geo }}$, $(\vec{m}, \vec{\phi})$ is in the region $\Theta_{\mathcal{E}}\left(\phi_{3}-\phi_{1}<2, \phi_{3}-\phi_{2}>1\right) \subset \mathrm{Stab}^{\text {nd }}$. 
We may consider the image $W$ of $\operatorname{Ker}\left(\Theta_{\mathcal{E}}\left(\phi_{3}-\phi_{1}<2, \phi_{3}-\phi_{2}>1\right)\right)$ on $\mathrm{P}\left(\mathrm{K}_{\mathbb{R}}\left(\mathbf{P}^{2}\right)\right)$ and the wall $\mu(E)=\mu(F)$. By similar arguments in Proposition 2.5 and the result of Lemma $1.16, W$ is connected and is a 'triangle' on the projective $\left\{1, \frac{\mathrm{ch}_{1}}{\mathrm{ch}_{0}}, \frac{\mathrm{ch}_{2}}{\mathrm{ch}_{0}}\right\}$-plane. On the $\left\{1, \frac{\mathrm{ch}_{1}}{\mathrm{ch}_{0}}, \frac{\mathrm{ch}_{2}}{\mathrm{ch}_{0}}\right\}$-plane, $W$ is the union of two regions bounded by $\left\{l_{E_{1} E_{2}}^{r}, l_{E_{2} E}, l_{E_{1} E}^{r}\right\}$ and $\left\{l_{E E_{1}}^{r}, l_{E_{2} E_{1}}^{r}\right\}$ respectively.

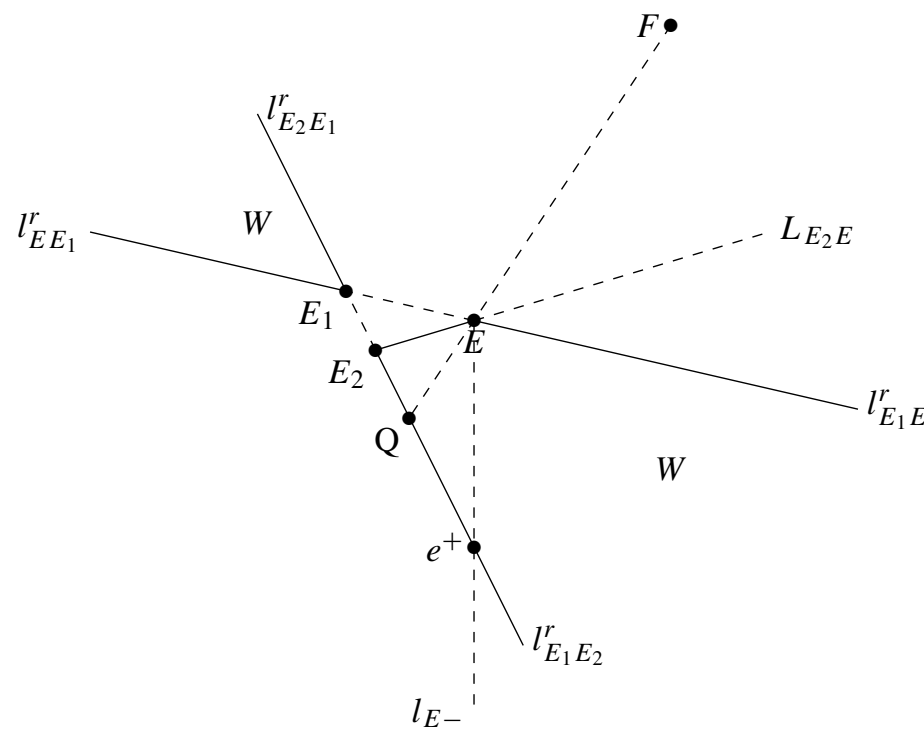

As $\mu\left(E_{1}\right)<\mu(F(-3)), F$ is above $L_{e_{1}^{+} e_{1}^{r} E_{2} E}$, on which $\chi\left(-, E_{1}\right)=0$. The ray $l_{F E}^{r}$ is in the angle spanned by $l_{E e_{1}^{+}}$and $l_{E-}$. Let $Q$ be the intersection of $L_{E F}$ and $L_{e_{3}^{+} e_{3}^{l} E_{2} E_{1}}$, then it is on the segment $l_{E_{2} e^{+}}$. By the position of the lines, $L_{E F} \cap W$ $=l_{E Q}$ and it is the only wall on which $\phi(F)=\phi(E)$. By Proposition 2.5 and Lemma 1.17, we have $\phi(E)<\phi(F)$, when $\operatorname{Ker} Z$ is in the triangle area $\operatorname{TR}_{E_{2}} Q E$; and we have $\phi(E)>\phi(F)$, when $\operatorname{Ker} Z$ is in $\mathrm{TR}_{E Q e^{+}}$. As $l_{E Q}$ is the only wall, in $\Theta_{\mathcal{E}}\left(\phi_{3}-\phi_{1}<2, \phi_{3}-\phi_{2}>1\right), \phi(E)<\phi(F)$ if and only if Ker $Z$ is in $\operatorname{TR}_{E_{2} Q E} \subset$ $\mathrm{Geo}_{L P}$. We get the contradiction that $F$ cannot be stable at any point in $\Theta_{E}^{-}$.

The $\Theta_{F}^{+}$part is proved in the same way.

Proposition 3.4 Let $E$ and $F$ be two exceptional bundles, then $\Theta_{E}^{+} \cap \Theta_{F}^{+}$is non-empty if and only if $E=F$. The same statement holds for $\Theta_{E}^{-}$and $\Theta_{F}^{-}$. In addition $\Theta_{E}^{+} \cap$ $\Theta_{F}^{-}=\phi$ for any $E$ and $F$.

Proof For any stability condition in $\Theta_{E}^{ \pm}, E$ is stable. Assuming $\mu(E)<\mu(F)$, by Lemma 3.3, we only need prove that $\Theta_{F}^{-} \cap \Theta_{E}^{+}$is empty.

When $\mu(E)+3>\mu(F)$, we may choose an exceptional triple $\mathcal{F}=\left\{F_{1}, F_{2}, F_{3}=\right.$ $F\}$ being an extension of $F$ such that $\mu\left(F_{1}\right)<\mu(E)$. Such triple exists due to the correspondence of triples of dyadic numbers and exceptional triples. By Lemma 3.3, $F_{1}$ is not stable in $\Theta_{E}^{+}$, the intersection $\Theta_{F}^{-} \cap \Theta_{E}^{+}$is empty.

When $\mu(E)+3=\mu(F)$, in other words, $E=F(-3)$, we may choose an exceptional triple $\mathcal{F}=\left\{F_{1}, F_{2}, F_{3}=F\right\}$ being an extension of $F$. There is another exceptional 
triple $\mathcal{E}=\left\{E_{1}=E, E_{2}=F_{1}, E_{3}=F_{2}\right\}$. Suppose $\Theta_{F}^{-}$intersects with $\Theta_{E}^{+}$at a point $(\vec{m}, \vec{\phi})$ in $\Theta_{\mathcal{E}, E}^{+}$.

Since $\operatorname{Hom}\left(F, E_{1}[2]\right)=\operatorname{Hom}(F, F(-3)[2])=\mathbb{C}$, we have $\phi(F)-\phi\left(E_{1}\right)<2$. But $\phi\left(F_{2}\right)-\phi(E)=\phi\left(E_{3}\right)-\phi(E)>1$, by definition, we must have $\phi(F)-\phi\left(F_{2}\right)<$ 1 , and this implies the stability is in $\Theta_{\mathcal{F}}^{\nabla} \subset \operatorname{Stab}^{\mathrm{Geo}}\left(\mathbf{P}^{2}\right)$, which is a contradiction.

The last case is when $\mu(E)+3<\mu(F)$. We may choose an exceptional triple $\mathcal{F}=$ $\left\{F_{1}, F_{2}, F_{3}=F\right\}$ being an extension of $F$ such that $\mu\left(F_{1}\right)>\mu(E)+3$. Again, such triple exists due to the correspondence of triples of dyadic numbers and exceptional triples. Let $\mathcal{E}=\left\{E_{1}=E, E_{2}, E_{3}\right\}$ be an extension of $E$ such that $\mu\left(E_{3}\right)<\mu\left(F_{1}\right)-3$.

Suppose $F_{i}$ 's are stable at a point $(\vec{m}, \vec{\phi})$ in $\Theta_{\mathcal{E}, E}^{+}$. Then we have $\operatorname{Hom}(F, E[2])=$ $\operatorname{Hom}(E, F(-3)) \neq 0$, hence $\phi(F)<\phi(E)+2$. Notice that $\phi\left(F_{1}\right)<\phi(F)$ by definition, we get $\phi\left(F_{1}\right)<\phi(E)+1$. On the other hand, $\operatorname{Hom}\left(E_{3}, F_{1}\right) \neq 0$ by the choice of the slopes, so $\phi\left(E_{3}\right)<\phi\left(F_{1}\right)$. Hence $\phi\left(E_{3}\right)<\phi(E)+1$, which implies the stability is not in $\Theta_{\mathcal{E}}$.

As a conclusion, $\Theta_{F}^{-} \cap \Theta_{E}^{+}$is empty when $E \neq F$.

Corollary 3.5 The union of geometric and algebraic stability conditions has the following decomposition:

$$
\begin{aligned}
& \operatorname{Stab}^{\mathrm{Geo}}\left(\mathbf{P}^{2}\right) \cup \operatorname{Stab}^{\mathrm{Alg}}\left(\mathbf{P}^{2}\right) \\
& =\operatorname{Stab}^{\mathrm{Geo}}\left(\mathbf{P}^{2}\right) \coprod\left(\coprod_{\text {E exc sheaves }} \Theta_{E}^{ \pm}\right) \bigsqcup\left(\coprod_{\mathcal{E} \text { exc triple }} l_{\Theta_{\mathcal{E}}^{\text {pure }}}\right) .
\end{aligned}
$$

We are now ready to show $\operatorname{Stab}^{\mathrm{Geo}}\left(\mathbf{P}^{2}\right) \cup \operatorname{Stab}^{\mathrm{Alg}}\left(\mathbf{P}^{2}\right)$ forms the whole connected component. To do this, we need to prove that $\operatorname{Stab}^{\mathrm{Geo}}\left(\mathbf{P}^{2}\right) \cup \operatorname{Stab}^{\mathrm{Alg}}\left(\mathbf{P}^{2}\right)$ has no boundary point. The following important result is from [19]: the boundary of finitely many $\Theta_{\mathcal{E}}$ is contained in $\mathrm{Stab}^{\mathrm{Alg}}$.

Theorem 3.6 (Theorem 4.7 in [19]) Let $\mathcal{E}$ be an exceptional triple, we have

$$
\partial \Theta_{\mathcal{E}} \subset \operatorname{Stab}^{\mathrm{Alg}} .
$$

To prove the main result, we also need the following description for details of the boundary of $\Theta_{E}^{ \pm}$.

Lemma 3.7 Let $E$ be an exceptional bundle, the boundary of $\Theta_{E}^{+}$(as well as $\Theta_{E}^{-}$) is contained in the union of the boundary of $\mathrm{Stab}^{\mathrm{Geo}}$ and the boundary of $\Theta_{\mathcal{E}}^{\text {pure }}$ for exceptional triples $\mathcal{E}$ that contain $E$ :

$$
\partial \Theta_{E}^{+} \subset \partial \operatorname{Stab}^{\mathrm{Geo}} \bigcup\left(\coprod_{\mathcal{E}: \text { exc triple contains } E} \partial \Theta_{\mathcal{E}}^{\text {pure }}\right)
$$

Proof Let $\sigma \in \mathrm{Stab}^{\dagger}$ be a point on the boundary of $\Theta_{E}^{ \pm}$. By Theorem 3.6, $\sigma$ belongs to $\Theta_{\mathcal{F}}$ for some exceptional triple $\mathcal{F}=\left\{F_{1}, F_{2}, F_{3}\right\}$. The point $\sigma$ is not in $\Theta_{\mathcal{F}}^{\text {Geo }}$ as 
else it has an open neighborhood in $\mathrm{Stab}^{\mathrm{Geo}}$. The point $\sigma$ is also not an inner point of $\Theta_{\mathcal{F}}^{\text {pure }}$, as else it has an open neighborhood such that the only stable objects are $F_{i}[n]$ for $i=1,2,3$ and $n \in \mathbb{Z}$. By Proposition 3.4, $\sigma$ is not in the inner point of $\Theta_{\mathcal{F}}^{ \pm}$. Hence, $\sigma$ is either on the boundary of $\Theta_{\mathcal{F}}^{\text {pure }}$ or the boundary between $\Theta_{\mathcal{F}}^{\text {Geo }}$ and $\Theta_{\mathcal{F}}^{ \pm}$.

Note that $\partial \Theta_{\mathcal{F}}^{\text {pure }}=\Theta_{\mathcal{F}}\left(\phi_{3}-\phi_{2}>\phi_{2}-\phi_{1}=1\right) \cup \Theta_{\mathcal{F}}\left(\phi_{2}-\phi_{1}>\phi_{3}-\phi_{2}=\right.$ 1) $\cup \Theta_{\mathcal{F}}\left(\phi_{3}-\phi_{2}=\phi_{2}-\phi_{1}=1\right)$, in which the third part is also contained in $\partial \Theta_{\mathcal{F}}^{\nabla}$ and hence $\partial \operatorname{Stab}^{\mathrm{Geo}}\left(\mathbf{P}^{2}\right)$. Suppose $\sigma$ is in $\Theta_{\mathcal{F}}\left(\phi_{2}-\phi_{1}>\phi_{3}-\phi_{2}=1\right)$, by a similar argument as that in Lemma 2.4, the only $\sigma$-semistable objects are $F_{1}[n]$ or in ${ }^{\perp} F_{1}$. By Proposition 3.1, for any exceptional bundle $E^{\prime}$ in ${ }^{\perp} E, E^{\prime}$ is $\sigma$-semistable. The object $F_{1}$ must be $E[n]$. Due to the same argument, $\sigma$ is not in $\Theta_{\mathcal{F}}\left(\phi_{3}-\phi_{2}>\phi_{2}-\phi_{1}=1\right)$. We may choose $\mathcal{F}$ such that $F_{1}=E$.

Proposition 3.8 The boundary of the geometric stability conditions is contained in the space of algebraic stability conditions:

$$
\partial \mathrm{Stab}^{\mathrm{Geo}} \subset \mathrm{Stab}^{\mathrm{Alg}} .
$$

Proof Let $\sigma=(Z, \mathcal{P})$ be a stability condition on $\partial \mathrm{Stab}^{\mathrm{Geo}}$.

I. $\sigma$ is non-degenerate.

By Lemma 1.14, Ker $Z$ is on the boundary of $\operatorname{Geo}_{L P}$. When $\operatorname{Ker} Z$ is at the infinity line of $\mathrm{P}\left(\mathrm{K}_{\mathbb{R}}\left(\mathbf{P}^{2}\right)\right)$, its locus is $(0,0,1)$ as this is the only asymptotic line of the parabola. However, $\sigma$ cannot be a stability condition, since $Z(k(x))=Z([0,0,1])$ $=0$, contradicting the fact that $k(x)$ is semistable on the boundary. When $\operatorname{Ker} Z$ is not at the infinity line, by Proposition 2.5, $\sigma$ is either on the boundary of $\Theta_{\mathcal{E}}$ for an exceptional triple $\mathcal{E}$ or on the $\bar{\Delta}_{\frac{1}{2}}$ but not between any $e^{r}$ and $e^{l}$. The first case is due to Theorem 3.6.

The second case is more complicated, we will show that $\sigma$ cannot satisfy the support condition. Let Ker $Z$ be $(1, s, q)$, then $q=\frac{1}{2} s^{2}-\frac{1}{2}$. Let $L_{1}$ be the line on the $\left\{1, \frac{\mathrm{ch}_{1}}{\mathrm{ch}_{0}}, \frac{\mathrm{ch}_{2}}{\mathrm{ch}_{0}}\right\}$-plane passing through the points $(1, s, q)$ and $\left(1, s-3, \frac{1}{2}(s-3)^{2}-\frac{1}{2}\right)$. Let $L_{2}$ be the line $\frac{\mathrm{ch}_{1}}{\mathrm{ch}_{0}}=s$. Let $D_{r}$ be the set of characters defined as

$D_{r}:=\left\{v \in \mathrm{K}\left(\mathbf{P}^{2}\right) \mid \bar{v}\right.$ is strictly below $L_{1}$ and to the right of $\left.L_{2},\|\bar{v}-(1, s, q)\|<r\right\}$.

Let $v_{n}$ be a character in $D_{\frac{1}{n}}$, as $v_{n}$ is below $L_{1}$, it is below the Le Potier curve. By the classification result of [14], $\mathfrak{M}_{M G}\left(v_{n}\right)$ is non-empty. Adopting the notation in [18], as $v_{n}$ is below $L_{1}$, it is in $\mathfrak{R}_{E}$ for exceptional $E$ with $\frac{\mathrm{ch}_{1}}{\operatorname{ch}_{0}}(E)<s$. By the criterion for the last wall in [12] or [18], the stability condition $\sigma$ is above the last wall of $v_{n}$, in another word, there are $\sigma$-stable objects with character $v_{n}$. On the other hand, as $\|\bar{v}-(1, s, q)\|<\frac{1}{n}$ and the $\operatorname{Ker} Z$ is $(1, s, q)$, we have

$$
\left|Z\left(v_{n}\right)\right| \leq \frac{1}{n}\left\|v_{n}\right\| .
$$

The stability condition $\sigma$ does not satisfy the support condition. We get the contradiction. 
II. $\sigma$ is degenerate.

By [8], $\operatorname{Stab}^{\dagger}\left(\mathbf{P}^{2}\right) \rightarrow \operatorname{Hom}_{\mathbb{Z}}\left(\mathrm{K}\left(\mathbf{P}^{2}\right), \mathbb{C}\right)$ is a local homeomorphism, the degenerate locus has codimension 2 in $\operatorname{Stab}^{\dagger}\left(\mathbf{P}^{2}\right)$. By [4] Proposition 3.3, the destabilizing wall $W_{P}^{k(x)}$ for the skyscraper sheaf is of codimension 1. As the destabilizing walls are locally finite, we may assume $\sigma$ is on the boundary of $W_{P}^{k(x)}$ for a character $P$ in $\mathrm{K}_{\mathbb{R}}\left(\mathbf{P}^{2}\right)$. By Lemma 1.16, the kernel of the central charge of any stability condition on $W_{P}^{k(x)} \cap \mathrm{Stab}^{n d}$ is on the line $L_{P k(x)}$, which is a line parallel to the $\frac{\mathrm{ch}_{2}}{\mathrm{ch}_{0}}$-axis on the $\left\{1, \frac{\mathrm{ch}_{1}}{\mathrm{ch}_{0}}, \frac{\mathrm{ch}_{2}}{\mathrm{ch}_{0}}\right\}$-plane. As the kernel of $W_{P}^{k(x)} \cap \mathrm{Stab}^{n d}$ has codimension one and is on the boundary of $\mathrm{Geo}_{L P}$, it is the segment of $l_{e e^{+}}$for some exceptional bundle $E$. $W_{P}^{k(x)} \cap \mathrm{Stab}^{n d}$ is contained in the closure of $\Theta_{\mathcal{E}}^{\mathrm{Geo}} \cup \Theta_{\mathcal{E}^{\prime}}^{\mathrm{Geo}}$ for any $\mathcal{E}=\left\{E_{1}, E_{2}, E\right\}$ and $\mathcal{E}^{\prime}=\left\{E, E_{2}^{\prime}, E_{3}^{\prime}\right\}$. Therefore, $\sigma$ is contained in the closure of $\Theta_{\mathcal{E}}^{\mathrm{Geo}} \cup \Theta_{\mathcal{E}^{\prime}}^{\mathrm{Geo}}$. By Theorem 3.6, $\sigma \in \operatorname{Stab}^{\mathrm{Alg}}\left(\mathbf{P}^{2}\right)$.

\subsection{Main result}

Theorem 3.9 The connected component $\operatorname{Stab}^{\dagger}\left(\mathbf{P}^{2}\right)$ in $\operatorname{Stab}\left(\mathbf{P}^{2}\right)$ that contains the geometric stability conditions is the union of geometric and algebraic stability conditions.

$$
\operatorname{Stab}^{\dagger}\left(\mathbf{P}^{2}\right)=\operatorname{Stab}^{\mathrm{Geo}}\left(\mathbf{P}^{2}\right) \bigcup \operatorname{Stab}^{\mathrm{Alg}}\left(\mathbf{P}^{2}\right)
$$

Proof We show that the boundary of $\bigsqcup_{E \text { exc sheaf }} \Theta_{E}^{ \pm}$and $\bigsqcup_{\mathcal{E} \text { exc triple }} \Theta_{\mathcal{E}}^{\text {pure }}$ is contained in $\operatorname{Stab}^{\mathrm{Geo}}\left(\mathbf{P}^{2}\right) \cup \operatorname{Stab}^{\dagger}\left(\mathbf{P}^{2}\right)$. Together with Corollaries 3.5 and 3.8, we get the conclusion.

Let $\sigma$ be a stability condition on the boundary of $\bigsqcup_{\mathcal{E} \text { exc triple }} \Theta_{\mathcal{E}}^{\text {pure }} . \sigma$ has at least three stable objects $A, B, C$ to generate the Grothendieck group of $\mathrm{D}^{b}\left(\mathbf{P}^{2}\right)$. There is an open neighborhood of $\sigma$ at where $A, B, C$ are always stable. Since the only stable objects in $\Theta^{\text {pure }}$ are $E_{i}[n]$ for $i=1,2,3$ and $n \in \mathbb{Z}, \sigma$ is on the boundary of at most one $\Theta^{\text {pure }}$. By Theorem 3.6, $\sigma$ is in $\mathrm{Stab}^{\mathrm{Alg}}$.

Let $\sigma$ be a stability condition on the boundary of $\bigsqcup_{E \text { exc sheaf }} \Theta_{E}^{ \pm}$, then for any open neighborhood $U$ of $\sigma, U$ intersect the union of boundaries of $\Theta_{E}^{q}$. Now by Lemma 3.7, $U$ intersects the union of $\partial \operatorname{Stab}^{\mathrm{Geo}}\left(\mathbf{P}^{2}\right)$ and $\partial \Theta_{\mathcal{E}}^{\text {pure }} . \sigma$ is on the boundary of either $\operatorname{Stab}^{\mathrm{Geo}}\left(\mathbf{P}^{2}\right)$ or $\bigsqcup_{\mathcal{E} \text { exc triple }} \Theta_{\mathcal{E}}^{\text {pure }}$. By Proposition 3.8 and the previous paragraph on the boundary of $\bigsqcup_{\mathcal{E} \text { exc triple }} \Theta_{\mathcal{E}}^{\text {pure }}, \sigma$ is in $\operatorname{Stab}^{\operatorname{Alg}}\left(\mathbf{P}^{2}\right)$.

Corollary 3.10 $\operatorname{Stab}^{\dagger}\left(\mathbf{P}^{2}\right)$ is contractible.

Proof Each $\Theta_{\mathcal{E}}^{\text {pure }}$ has an open neighborhood in $\Theta_{\mathcal{E}}$ which does not intersect any other $\Theta_{\mathcal{E}^{\prime}}^{\text {pure }}$. For example, one may choose the region of $\phi_{3}-\phi_{2}>\frac{1}{2}$ and $\phi_{2}-\phi_{1}>\frac{1}{2}$ in $\Theta_{\mathcal{E}}$. As $\operatorname{Stab}^{\dagger}$ admits a metric, we may then choose open neighborhoods of $\Theta_{\mathcal{E}}^{\text {pure, }}$ s which do not intersect with each other. By Corollary 3.5, $\operatorname{Stab}^{\dagger}\left(\mathbf{P}^{2}\right)$ is homotopic to its subspace $\operatorname{Stab}^{\mathrm{Geo}}\left(\mathbf{P}^{2}\right) \bigsqcup\left(\coprod_{E \text { exc sheaves }} \Theta_{E}^{ \pm}\right)$. Each $\Theta_{E}^{+}$has an open neighborhood in $\operatorname{Stab}^{\mathrm{Geo}}\left(\mathbf{P}^{2}\right) \bigsqcup\left(\bigsqcup_{E \text { exc sheaves }} \Theta_{E}^{ \pm}\right)$which does not intersects any other $\Theta_{E}^{ \pm}$. For example, we may choose any exceptional triple $\mathcal{E}$ with $E_{1}=E$ and take $\Theta_{\mathcal{E}}^{\mathrm{Geo}} \cup$ 
$\Theta_{E}^{+}$as the open neighborhood. We may contract $\operatorname{Stab}^{\mathrm{Geo}}\left(\mathbf{P}^{2}\right) \bigsqcup\left(\coprod_{E \text { exc sheaves }} \Theta_{E}^{ \pm}\right)$to $\mathrm{Stab}^{\mathrm{Geo}}$ which is a contractible space.

Acknowledgements The author is grateful to Arend Bayer, Zheng Hua, Yu Qiu and Xiaolei Zhao for helpful conversations. The author appreciates the referee's suggestions, in particular, the shortcut proofs for Proposition 3.1 and 3.4 are due to the referee. The author is supported by ERC starting Grant No. 337039 "WallXBirGeom".

Open Access This article is distributed under the terms of the Creative Commons Attribution 4.0 International License (http://creativecommons.org/licenses/by/4.0/), which permits unrestricted use, distribution, and reproduction in any medium, provided you give appropriate credit to the original author(s) and the source, provide a link to the Creative Commons license, and indicate if changes were made.

\section{References}

1. Arcara, D., Bertram, A., Coskun, I., Huizenga, J.: The minimal model program for the Hilbert scheme of points on $\mathbb{P}^{2}$ and Bridgeland stability. Adv. Math. 235, 580-626 (2013)

2. Auroux, D., Katzarkov, L., Orlov, D.: Mirror symmetry for del Pezzo surfaces: vanishing cycles and coherent sheaves. Invent. Math. 166(3), 537-582 (2006)

3. Bayer, A., Bridgeland, T.: Derived automorphism groups of K3 surfaces of Picard rank 1. arXiv:1310.8266, to appear in Duke Math. J

4. Bayer, A., Macrì, E.: The space of stability conditions on the local projective plane. Duke Math. J. 160(2), 263-322 (2011)

5. Bayer, A., Macrì, E., Stellari, P.: Stability conditions on abelian threefolds and some Calabi-Yau threefolds. arXiv: 1410.1585

6. Beřlinson, A.A.: The derived category of coherent sheaves on $\mathbf{P}^{n}$. Selecta Math. Soviet. 3(3),233-237 (1983/84). Selected translations

7. Bertram, A., Steffen, M., Wang, J.: The stability manifolds of $\mathbb{P}^{1}$ and local $\mathbb{P}^{1}$. Contemp. Math. 647, $1-17$ (2015)

8. Bridgeland, T.: Stability conditions on triangulated categories. Ann. Math. (2) 166(2), 317-345 (2007)

9. Bridgeland, T.: Stability conditions on $K 3$ surfaces. Duke Math. J. 141(2), 241-291 (2008)

10. Bridgeland, T.: Spaces of stability conditions. Proc. Sympos. Pure Math. 80, 1-21 (2009)

11. Bridgeland, T., Qiu, Y., Sutherland, T.: Stability conditions and the $\mathbf{A}_{2}$ quiver. arXiv:1406.2566

12. Coskun, I., Huizenga, J., Woolf, M.: The effective cone of the moduli space of sheaves on the plane. arXiv:1401.1613

13. Dimitrov, G., Katzarkov, L.: Bridgeland stability conditions on the acyclic triangular quivers. Adv. Math. 288, 825-886 (2016)

14. Drezet, J.-M., Le Potier, J.: Fibrés stables et fibrés exceptionnels sur $P_{2}$. Ann. Sci. École Norm. Sup. (4) 18(2), 193-243 (1985)

15. Gorodentsev, A.L., Rudakov, A.N.: Exceptional vector bundles on projective spaces. Duke Math. J. 54(1), 115-130 (1987)

16. Ikeda, A.: Stability conditions on $\mathrm{CY}_{\mathrm{N}}$ categories associated to $\mathbf{A}_{n}$-quivers and period maps. arXiv: 1405.5492

17. Le Potier, J.: Lectures on vector bundles. Volume 54 of Cambridge Studies in Advanced Mathematics. Cambridge University Press, Cambridge (1997). (Translated by A. Maciocia)

18. Li, C., Zhao, X.: Birational models of moduli spaces of coherent sheaves on the projective plane. arXiv: 1603.05035

19. Macrì, E.: Some examples of spaces of stability conditions on derived categories. arXiv:math/0411613

20. Macrì, E.: Stability conditions on curves. Math. Res. Lett. 14(4), 657-672 (2007)

21. Okada, S.: Stability manifold of $\mathbb{P}^{1}$. J. Algebr. Geom. 15(3), 487-505 (2006)

22. Orlov, D.O.: Projective bundles, monoidal transformations, and derived categories of coherent sheaves. Izv. Ross. Akad. Nauk Ser. Mat. 56(4), 852-862 (1992)

23. Qiu, Y.: Stability conditions and quantum dilogarithm identities for Dynkin quivers. Adv. Math. 269, 220-264 (2015)

24. Qiu, Y., Woolf, J.: Contractible stability spaces and faithful braid group actions, (2014). arXiv:1407.5986 